\title{
The dentist who sat on her chair and lost a leg. N2O?
}

GIOVANNI LANDONI ${ }^{1,2}$, PASQUALE NARDELLI ${ }^{1}$

${ }^{1}$ Department of Anesthesia and Intensive Care, IRCCS San Raffaele Scientific Institute, Milan, Italy

${ }^{2}$ Vita-Salute San Raffaele University, Milan, Italy

Corresponding author:

Giovanni Landoni

IRCCS San Raffaele Scientific Institute

Department of Anesthesia and Intensive Care

Via Olgettina 60, 20132 Milan, Italy

Phone: +3902 26434524

Fax: +390226436152

E-mail: landoni.giovanni@hsr.it

\section{ABSTRACT}

A 35-year-old female dentist laid on her chair to test an $\mathrm{N} 2 \mathrm{O}$ machine, and after only a few minutes of inhaling the $\mathrm{N} 2 \mathrm{O}$, she developed acute pyramidal syndrome. The patient started walking again eight months later, but still suffers from lower limb motor deficit, in spite of intensive rehabilitation. Genetic tests later showed that the patient had Type 3 homocystinuria.

This is the first case report of acute neurological toxicity after brief administration of N2O. We suggest starting vitamin B12 and folic acid supplements promptly in patients who experience neurological symptoms after receiving $\mathrm{N} 2 \mathrm{O}$.

Key words: case report, neurological toxicity, nitrous oxide, adverse reaction, homocystinuria, dental care

\section{INTRODUCTION}

A 35-year-old dentist, from Italy, breathed $\mathrm{N}_{2} \mathrm{O}$ for a few minutes, resulting in permanent motor deficit, in spite of intensive rehabilitation. Written consent has been obtained from the patient to share her story.

\section{CASE REPORT}

The patient is a young woman, of prior good health, who exercised regularly (running, skiing) and led an orderly life. In 2001, the patient was hospitalized with vertigo and paresthesia in her right leg. An MRI was performed, uncovering a left fronto-parietal lesion, possibly secondary to a monophasic inflammatory process. The etiology of the episode remained cryptic, with a temporal association to a flu shot received two days before the on- set of symptoms. The episode completely resolved, resulting in no motor or mental deficits.

In 2010, the woman laid on her dentistry chair to test, on herself, an N2O machine she was about to buy. The technician from the company was administering the $\mathrm{N} 2 \mathrm{O}$, to prove the safety and ease of use of the machine. After only a few minutes, the dentist started experiencing neurological symptoms, which evolved into an acute right pyramidal syndrome, involving the entire right half of the body. After 20 minutes, the patient was hospitalized and investigated for possible stroke. However, CT and MRI showed no changes from the previous examination, still describing the abovementioned fronto-parietal lesion.

The patient improved slightly after a few days, and after daily physiotherapy started walking again eight months later, but - as of today (6 years after the episode) - she still suffers from right lower limb motor deficit and ulnar nerve hypoesthesia, in spite of intensive rehabilitation and physical therapy.

Differential diagnosis included: equipment failure (with delivery of a hypoxic mixture), air embolism (with concomitant right to left atrial shunt), pre-existing vitamin B12 or folate deficiency, psychiatric disorder, autosomal recessive methylene tetrahydrofolate reductase (MTHFR) deficiency or polymorphism.

The N2O machine was tested to rule out malfunctioning and the occurrence of hypoxic flows, and was found to be working properly.

Air embolism associated with a patent foramen ovale was considered, since nitrous oxide expands existing gas bubbles which could then pass into the central nervous system. (1) However, echocardiography did not document a right to left shunt in our patient, and the patient had no source of air embolism (e.g. no intravenous line). Pre-existing vitamin B12 or folate deficiency has been associated with the occurrence of neurological events in nitrous oxide abusers, (2) but it has also been reported in patients who underwent single-time prolonged nitrous oxide anesthesia. (3) The patient did not have symptoms of vitamin $\mathrm{B} 12$ or folate deficiency before the "nitrous oxide incident" and the laboratory exams were completely normal upon hospital admission.

Psychiatric disorders (e.g. Munchausen syndrome) were ruled out.

Genetic tests were performed two years after the episode and showed that the patient had Type 3 homocystinuria due to a homozygote C677T mutation of the MTHFR gene. This mutation leaves approximately $50 \%$ residual enzyme activity and marks enzyme lability to heat inactivation. (4) It is associated with a cytosine to thymine mutation at nucleotide position 677 , encoding for an alanine-223 to valine substitution. This mutation is quite common, with a homozygote frequency up to $17 \%$ in the Caucasian population. Carrier frequency may be as high as $40 \%$. (4)

The most plausible hypothesis is that nitrous oxide was the cause of the acute neurological deficit. It is possible, in fact, that the encephalitis triggered a neurological remodeling with the development of alternate neural networks - which allowed the patient to have no clinical sequelae despite structural abnormalities. Nitrous oxide has many central effects mediated by N-methyl-D-aspartate (NMDA) receptors, $\alpha$-amino-3-hydroxy-5-methyl-4isoxazolepropionic acid receptor (AMPA) receptors, neuronal nicotinic receptors, 2-pore domain potassium channels, involving adrenergic, gabaergic and opioidergic pathways. (5) Therefore, it is not inconceivable that one of these actions 
somehow interrupted the compensatory neural pathways she had developed. This is, however, merely speculative, and has never been described previously.

\section{DISCUSSION}

Nitrous oxide is widely used as an analgesic-anesthetic drug. The large ENIGMAII trial (6) proved nitrous oxide as safe in patients known or suspected to have coronary artery disease undergoing major, non-cardiac surgery.

Because of its psychoactive properties, it is well-known for its abuse potential. Its side effects include limb numbness, formication and muscular weakness. Severe neurological side effects have been observed in nitrous oxide abusers, with progressive demyelination and consequent axonal lesions of peripheral nerves. (7) A large questionnaire study, interviewing around
35,000 dentists and assistants, demonstrated that chronic professional exposure to nitrous oxide relates to polyneuropathy 3-to-4-fold when compared to the general population. (8) Nonetheless, $\mathrm{N} 2 \mathrm{O}$ is the most frequently used agent for sedation in dental procedures.

Many studies demonstrated that $\mathrm{N} 2 \mathrm{O}$ selectively inhibits methionine synthase, a vitamin B12 dependent enzyme which plays a key role in the folate cycle. (9) The folate cycle is fundamental for the production of DNA, myelin and catecholamines. When $\mathrm{N} 2 \mathrm{O}$ is cleaved in azote and oxygen, it causes vitamin B12 oxidation and therefore the impossibility for it to act as a coenzyme. Another key enzyme in the folate cycle is methylene tetrahydrofolate reductase (MTHFR). (9)

In the presence of a genetic deficit of MTHFR, a simultaneous iatrogenic impairment of methionine synthase could result in a complete block of the folate cycle, with ill-fated effects on the production of myelin and DNA.

This is the first reported case of neurological damage triggered by a very short exposure to nitrous oxide in an adult patient. Notably, Selzer et al. (10) described the death of a child who underwent nitrous oxide anesthesia twice and was later diagnosed with MTHFR deficiency. The autopsy showed asymmetric cerebral atrophy and severe demyelination.

Millions of patients are receiving nitrous oxide every year, not only for dental care, but also in general surgery. We suggest nitrous oxide not be given to patients with previous neurological insults. We also suggest that patients with neurological insults after nitrous oxide administration receive immediate supplementation with vitamins and folic acid and undergo a MTHFR gene mutation analysis.

\section{REFERENCES}

1. Benavides R, Maze M, Franks NP. Expansion of gas bubbles by nitrous oxide and xenon. Anesthesiology 2006;104:299-302.

2. Massey TH, Pickersgill TT, J Peall K. Nitrous oxide misuse and vitamin B12 deficiency. BMJ Case Rep 2016 May 31;2016. pii: bcr2016215728. doi: 10.1136/bcr-2016-215728.

3. Hadzic A, Glab K, Sanborn KV, Thys DM. Severe neurologic deficit after nitrous oxide anesthesia. Anesthesiology 1995;83:863-6.

4. Deloughery TG1, Evans A, Sadeghi A, McWilliams J, Henner WD, Taylor LM Jr, Press RD. Common mutation in methylenetetrahydrofolate reductase: correlation with homocysteine metabolism and late-onset vascular disease. Circulation 1996;94:3074-3078

5. Emmanouil DE, Quock RM. Advances in understanding the actions of nitrous oxide. Anesth Prog 2007;54:9-18.

6. Myles PS, Leslie K, Chan MT, Forbes A, Peyton PJ, Paech MJ, Beattie WS, Sessler DI, Devereaux PJ, Silbert B, Schricker T, Wallace S; ANZCA Trials Group for the ENIGMA-II investigators. The safety of addition of nitrous oxide to general anaesthesia in at-risk patients having major non-cardiac surgery (ENIGMA-II): a randomised, single-blind trial. Lancet 2014;384:1446-54.

7. Brodsky JB, Cohen EN, Brown BW Jr, Wu ML, Whitcher CE. Exposure to nitrous oxide and neurologic disease among dental professionals. Anesth Analg 1981;60:297-301.

8. Intercollegiate Advisory Committee on Sedation in Dentistry. Standards for Conscious Sedation in the Provision of Dental Care, 2015. Available at: https://www.rcseng.ac.uk/fds/Documents/dental-sedation-report-2015-web-v2.pdf. Accessed on June 17 th, 2016.

9. Nagele P, Brown F, Francis A, Scott MG, Gage BF, Miller JP; VINO Study Team. Influence of nitrous oxide anesthesia, B-vitamins, and MTHFR gene polymorphisms on perioperative cardiac events: the vitamins in nitrous oxide (VINO) randomized trial. Anesthesiology 2013;119:19-28.

10. Selzer RR, Rosenblatt DS, Laxova R, Hogan K. Adverse effect of nitrous oxide in a child with 5,10-methylenetetrahydrofolate reductase deficiency. N Engl J Med 2003;349:45-50. 